\title{
In vitro Self-Assembly of Human Pericyte-Supported Endothelial Microvessels in Three-Dimensional Coculture: A Simple Model for Interrogating Endothelial-Pericyte Interactions
}

\author{
J.P. Waters ${ }^{c} \quad$ M.S. Kluger ${ }^{a} \quad$ M. Graham ${ }^{b} \quad$ W.G. Chang ${ }^{\text {a }} \quad$ J.R. Bradley ${ }^{c}$ \\ J.S. Pober ${ }^{\mathrm{a}}$ \\ ${ }^{a}$ Department of Immunobiology and ${ }^{\mathrm{b}}$ Center for Cellular and Molecular Imaging, Yale University School of \\ Medicine, New Haven, Conn., USA; ' Department of Medicine, University of Cambridge, Addenbrooke's Hospital, \\ Cambridge, UK
}

\section{Key Words}

Vasculogenesis - Three-dimensional protein matrix .

Pericyte $\cdot$ Endothelial cells $\cdot$ Coculture

\begin{abstract}
We describe a method for coculture of macro- or microvascular human endothelial cells (ECs) and pericytes (PCs) within a 3-dimensional (3-D) protein matrix resulting in lumenized EC cords invested by PCs. To prevent apoptotic cell death of ECs in 3-D culture, human umbilical vein or dermal microvascular ECs were transduced to express the antiapoptotic protein $\mathrm{BCl}-2$. To prevent $\mathrm{PC}$-mediated gel contraction, the collagen-fibronectin gel was polymerized within a polyglycolic acid nonwoven matrix. Over the first 24-48 h, EConly gels spontaneously formed cords that developed lumens via vacuolization; such vascular networks were maintained for up to 7 days. In EC-PC cocultures, PCs were recruited to the EC networks. PC investment of EC cords both limited the lumen diameter and increased the degree of vascular network arborization. Peg and socket junctions formed between ECs and PCs in this system, but dye transfer, indicative of gap junction formation, was not observed. This simple system can be used to analyze bidirectional signals between ECs and PCs in a 3-D geometry.

(c) 2013 S. Karger AG, Basel
\end{abstract}

\section{Introduction}

Aberrations in endothelial cell (EC)-pericyte (PC) interactions are a feature of many vascular diseases ranging from the PC 'drop out' of diabetic retinopathy [1], hereditary hemorrhagic telangiectasia [2] and several tumors [3] to the proliferation of the mesangial cells in glomerular disease [4-6] and ectopic recruitment of PCs to lymphatics in lymphedema distichiasis [7]. While insights into the molecular mechanisms of the interaction between the two cell types have accumulated over the last 5 years, many questions remain as to the physiology and pathology of PCs and their interaction with ECs, answers to which may lead to therapeutics for such vascular pathologies, as well as guide tissue engineering of microvessels for repair of damaged organs. As many behaviors of vascular cells are species specific, it is important to develop assays using human cells. Our laboratory has previously reported a system in which mixtures of human umbilical vein (HUV)ECs and human microvascular PCs can be suspended in a 3-dimensional (3-D) collagen-fibronectin protein matrix using a nonwoven polyglycolic acid (PGA) scaffold to prevent PC-mediated gel contraction. The gel may then be subcutaneously implanted into an immunodeficient SCID beige mouse host, after which

\section{KARGER}

E-Mail karger@karger.com

www.karger.com/jvr
(C) 2013 S. Karger AG, Basel

1018-1172/13/0504-0324\$38.00/0
Dr. John P. Waters

Department of Medicine, University of Cambridge

Level 5 (Box 157), Addenbrooke's Hospital, Hills Road

Cambridge, CB2 0QQ (UK)

E-Mail jpw55@cam.ac.uk 
the implanted vascular cells organize to form human microvessels that become perfused following spontaneous inosculation with host microvessels [8]. In this report, we demonstrate that when the ECs are transduced to express the antiapoptotic protein $\mathrm{Bcl}-2$, this same 3-D system can be used for extended coculture of ECs and PCs in vitro during which the ECs form lumenized networks that recruit PCs and are themselves modified by the interaction.

\section{Materials and Methods}

\section{Cell Isolation and Transduction of ECs}

HUVECs and human placental PCs were each isolated and cultured as described $[9,10]$ or, alternatively in the case of HUVECs, purchased (Lonza, Walkersville, Md., USA). Human dermal microvascular endothelial cells (HMVECs) were purchased from the same vendor (Lonza). All cell types were used before passage 6. A retroviral vector encoding the D34A caspase-resistant form of Bcl2 was used to stably transduce primary cultures of the ECs as previously described [11]; the percentage of cells expressing Bcl-2 was $>95 \%$ as assessed by intracellular staining and FACS analysis (data not shown). For fluorescence microscopic analyses, ECs and PCs were labeled with PKH67 green fluorescence lipophilic membrane dye and PKH26 red fluorescence lipophilic membrane dye (both from Sigma-Aldrich, Saint Louis, Mo., USA), respectively, as per the manufacturer's instructions. To label PCs for identification by transmission electron microscopy, we utilized Molday ION Rhodamine B (BioPAL, Worcester, Mass., USA), an ultrasmall paramagnetic iron oxide nanoparticle (USPIO) of $35 \mathrm{~nm}$ diameter that is positively charged for enhanced cellular uptake, as described by others [12,13]. In brief, 100,000 PCs were plated in the well of a 24-well tissue culture plate in $0.5 \mathrm{ml}$ of M199 containing 20\% FBS, cultured overnight and then incubated for an additional $16 \mathrm{~h}$ under standard growth conditions $\left(5 \% \mathrm{CO}_{2}\right.$, at $\left.37^{\circ} \mathrm{C}\right)$ with $200 \mu \mathrm{g} / \mathrm{ml}$ of Molday ION Rhodamine B USPIOs, a concentration permitting optimal uptake by PCs as judged by Rhodamine B fluorescence. PCs were then rinsed twice with phosphate-buffered saline supplemented with $0.1 \mathrm{~g} / \mathrm{l} \mathrm{Ca}^{2+}$ and $0.1 \mathrm{~g} / \mathrm{Mgg}^{2+}$ before incorporation into 3-D cultures.

\section{Formation of 3-D Cultures}

ECs, with or without PCs, were suspended in a solution of rat tail type 1 collagen $(1.5 \mathrm{mg} / \mathrm{ml}$; BD, Franklin Lakes, N.J., USA) and human plasma fibronectin $(90 \mu \mathrm{g} / \mathrm{ml}$; Millipore, Billerica, Mass., USA) in $25 \mathrm{mM} \mathrm{HEPES}$ and $1.5 \mathrm{mg} / \mathrm{ml} \mathrm{NaHCO} 3$ buffered M199 medium at $4^{\circ} \mathrm{C}$, and the $\mathrm{pH}$ was adjusted to 7.4 using $0.1 \mathrm{M} \mathrm{HCl}$. The suspension was then pipetted into 48 -well tissue culture plates in a volume of $350 \mu \mathrm{l}$ per well with a final cell number of $1.5 \times 10^{6}$ ECs \pm 40,000 PCs. A non-woven PGA mesh (Concordia Fibers, Coventry, R.I., USA) was placed within the middle of the suspension, and the temperature was raised to $37^{\circ} \mathrm{C}$ in $5 \% \mathrm{CO}_{2}$-air for 20 min to allow polymerization of the protein gel. The gel was then overlaid with $0.5 \mathrm{ml}$ of medium [for HUVECs: M199 with endothelial growth supplement (50 $\mu \mathrm{g} / \mathrm{ml}$; Sigma-Aldrich) and 20\% FBS; for HMVECs: Clonetics EGM-2-MV medium (Lonza)], which was changed every second day. The 3-D cultures were maintained for 7 days.

\section{Imaging of 3-D Cultures}

The cultures were followed in real time using an Axiovert 200 M Carl Zeiss microscope by phase contrast microscopy and, in experiments using PKH dye-labeled cells, by fluorescence microscopy. In experiments with unlabeled cells, staining was performed on paraformaldehyde-fixed whole mounts of the gel or on 14- $\mu \mathrm{m}$ thick cryosections at specified time points. PCs were identified by staining with mouse anti-human NG2 primary antibody (ab83508; Abcam, Cambridge, UK) and Alexa Fluor ${ }^{\circledR}$ 594-labeled goat antimouse secondary antibody (Invitrogen, Life Technologies, Grand Island, N.Y., USA), and ECs were identified by staining with fluorescein-labeled Ulex europaeus agglutinin I (Vector Laboratories, Burlingame, Calif., USA). Nuclei were counterstained with 4',6-diamidino-2-phenylindole (DAPI). For electron microscopy, the matrices were washed in phosphate-buffered saline with $\mathrm{Ca}^{2+}$ and $\mathrm{Mg}^{2+}$, processed in fixative [ $2 \%$ paraformaldehyde, $2.5 \%$ glutaraldehyde in $0.1 \mathrm{M}$ sodium cacodylate buffer, $\mathrm{pH} 7.4$ (Electron Microscopy Sciences, Hatfield, Pa., USA)] for $1 \mathrm{~h}$ at room temperature, then stored at $4^{\circ} \mathrm{C}$ in cacodylate buffer until processing. To process further, samples were postfixed in $1 \%$ osmium tetroxide in cacodylate buffer for $1 \mathrm{~h}$ at room temperature, followed by 1 rinse in cacodylate buffer, then 3 rinses in $50 \mathrm{~mm}$ sodium maleate buffer, pH 5.2. Samples were then en bloc stained with $2 \%$ uranyl acetate in the same maleate buffer for $1 \mathrm{~h}$ while protected from light, rinsed in distilled water 3 times and then dehydrated through a graded ethanol series. Following 3 rinses of propylene oxide, the specimens were embedded in Epon 812 and baked at $60^{\circ} \mathrm{C}$ overnight. Semi-thin (100 nm thick) and thin $(60 \mathrm{~nm}$ thick) sections were then cut with a Leica UltraCut UCT microtome and further stained with contrast reagents (either toluidine blue or $2 \%$ uranyl acetate/lead citrate, respectively). USPIO-tagged PCs were identified in toluidine blue-stained semi-thin sections by light microscopy, and uranyl acetate-stained thin sections were subsequently viewed in a Tecnai 12 BioTwin transmission electron microscope at $80 \mathrm{kV}$. Images were taken using Morada CCD and iTEM (Olympus, Center Valley, Pa., USA) software.

Assessment of Cytoplasmic Dye Transfer between ECs and PCs PCs were prelabeled with $2^{\prime}, 7^{\prime}$-bis(2-carboxyethyl)-5(6)-carboxyfluorescein tetrakis(acetoxymethyl) ester (BCECF-AM; Sigma-Aldrich) before seeding in a gel. To do so, PCs were washed with Hank's solution and then exposed to BCECF-AM $(10 \mu \mathrm{M})$ for $40 \mathrm{~min}$ at $37^{\circ} \mathrm{C}$ in $5 \% \mathrm{CO}_{2}$-air. The BCECF-AM was then removed and the cells washed with Hank's solution before trypsinization for seeding into gels. ECs were labeled with PKH26 (Sigma-Aldrich) red fluorescence lipophilic membrane dye as described above. The gels were then assessed by fluorescence microscopy for costaining with ECs. The reverse labeling (ECs labeled with BCECF-AM and PCs with PKH26) was also carried out.

\section{Quantification and Statistical Analysis}

Quantification of cord networks within the 3-D matrix was carried out by taking random images through the matrix with phase contrast or fluorescence microscopy at low power using an Axiovert $200 \mathrm{M}$ Carl Zeiss microscope. The images were then analyzed using Image J software to quantify cord length (total and average length), branching points (nodes), tube width and cord number. Differences between treatments were analyzed using an unpaired two-tailed Student $t$ test. 

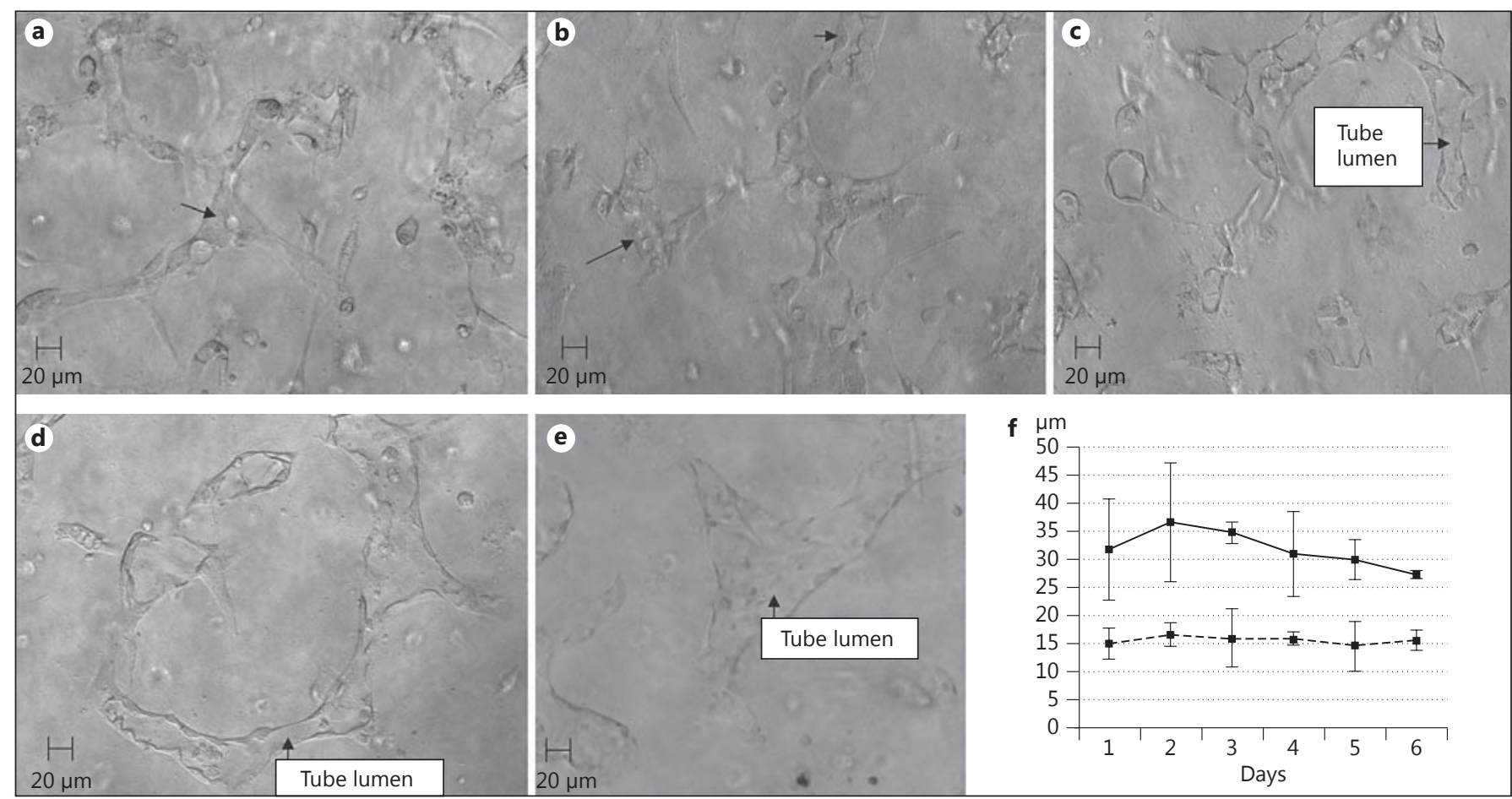

Fig. 1. Lumen formation by HUVEC in 3-D matrix gels in vitro. Phase contrast micrographs through the 3-D matrix, without a PGA mesh, containing Bcl-2-transduced HUVEC only at $17 \mathrm{~h} \mathrm{(a),}$ $24 \mathrm{~h}$ (b), $48 \mathrm{~h} \mathrm{(c)}$, and day 4 (d) and day 6 (e). Vacuole-type areas (arrow, a) can be seen lining up (long arrow, b) and coalescing from $24 \mathrm{~h}$ on (short arrow, b). From then, qualitatively, a progressive increase in lumen diameter is seen (c-e). $\mathbf{f}$ Quantitative analysis of tube diameter $(\mathrm{n}=3 ; \mathrm{p}<0.01$ for each time point). Black line = HUVEC only; dashed line = HUVEC and PC coculture. Error bars are standard deviations.

\section{Results}

As assessed by phase contrast microscopy, untransduced HUVECs cultured in a 3-D matrix spontaneously assemble into cord-like structures that break down and disintegrate by $24-36 \mathrm{~h}$ as a consequence of apoptotic cell death (data not shown). However, ECs transduced to express Bcl-2 do not undergo apoptosis in the first $24 \mathrm{~h}$ under these conditions of culture. Suspensions of Bcl-2transduced ECs still assemble into cords over the first $24 \mathrm{~h}$, but these structures persist and, over the next several days, form central vacuoles which coalesce to form lumens within the cords. Qualitatively, these lumens progressively increase in diameter over several days (fig. 1ae). However, by day 3 , some of the larger lumens appeared to expand to the point that it was not possible to unambiguously conclude that the cells on either side of the 'lumen' were still part of the same structure. We excluded these structures from our analyses and, as a result, we consciously underestimated the true mean lumen diam- eter at later times. As a result, the lumen diameter appears to decrease from day 3 onwards.

When PCs are included in the gel, Bcl-2-transduced ECs again form a network of cords over the same time frame and then undergo a similar process of vacuolization resulting in lumen formation. However, neither the cords nor their lumens achieved a diameter as great as that seen with EC-only culture (fig. 1f). This maintenance of narrow-diameter tubular structures was statistically significant on each day of culture and occurred with input cell numbers as few as 40,000 PCs compared to $1.5 \times 10^{6}$ ECs, about a 1:37 input cell ratio (fig. 1f). PCs appear to increase in number during the course of the experiment, reducing this ratio. Cultures initiated with greater PC numbers, i.e. above 40,000 , showed evidence of rapid PC overgrowth and could not be analyzed.

Microscopy of fluorescently labeled living cells within intact gels reveals that PCs are recruited to and then remain associated with the EC cords (fig. 2b). Coverage of the cords by PCs is not uniform but PC processes can be 
Fig. 2. Effect of PC coculture on lumen formation. a Phase contrast micrographs through the 3-D matrix, containing Bcl2-transduced HUVEC and PCs at day 3. b Live fluorescence microscopy through the 3-D matrix. Cells labeled with fluorescent membrane tracker HUVEC (green) and PCs (red).
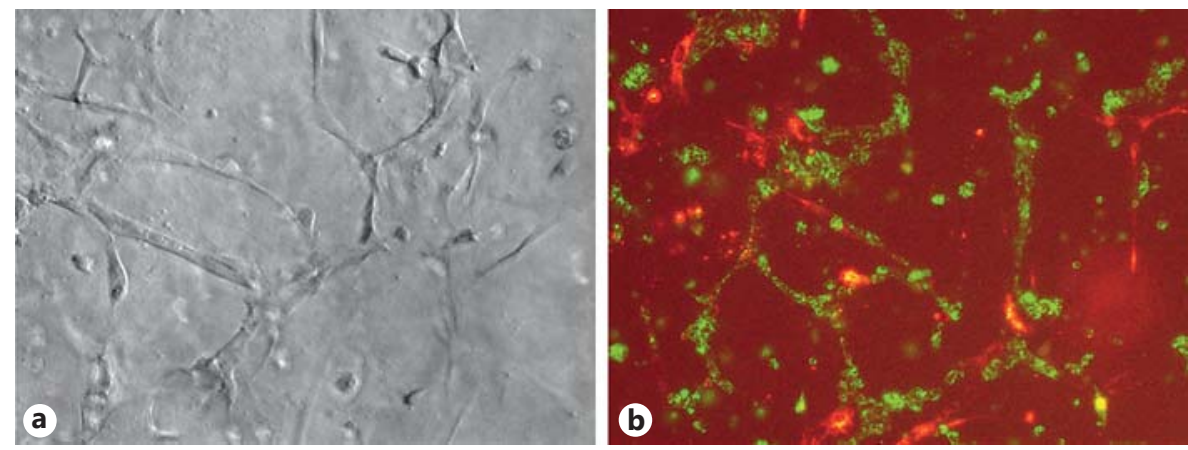

Fig. 3. Preferential association by PC with tubes formed by EC. Immunostaining of $14-\mu \mathrm{m}$-thick frozen sections of the 3-D matrix: Ulex europaeus agglutinin I (green), NG2 antibody (red) and DAPI (blue). a Low-power view showing HUVEC cords and PC association with enlargement (inset). b Lumen shown on cross-section of HUVEC cords. c High-power view of PC interaction with HUVEC cord.

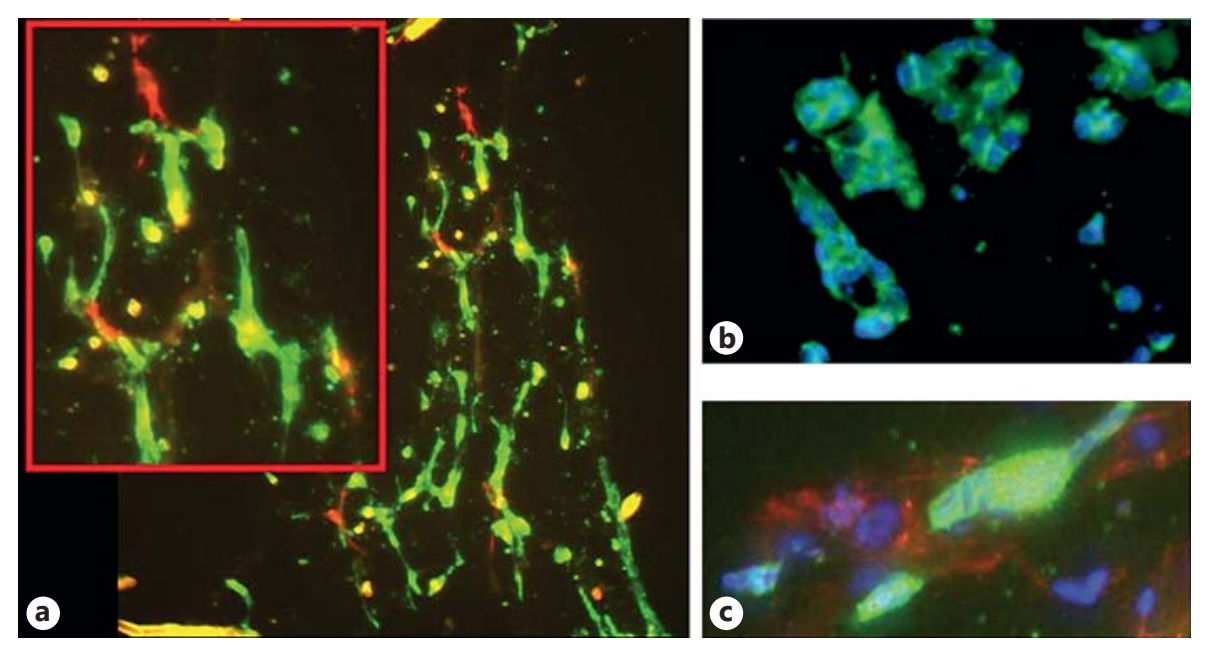

seen extending around EC cords (fig. 3a, c). The PGA scaffold required to prevent $\mathrm{PC}$-mediated contraction of the gel is autofluorescent, complicating the assessment of interactions solely by epifluorescence microscopy. To avoid this complication and to enhance image resolution of the cellular structure, we utilized immunohistochemical staining of thick cryosections. The rigidity given to the matrix by the PGA mesh is advantageous for the preparation of such sections as it allowed the matrix to be lifted out of the well with minimal distortion. Immunohistochemical staining of sections confirmed that PCs associate with the EC cords (fig. 3a), and the greater resolution of this technique confirmed that PCs extended processes around the EC cords (fig. 3c). The narrow lumen formed by the EC cords could also be visualized by this approach (fig. 3b). Morphometric analyses revealed that the recruitment of PCs also increases the arborization of the vascular network that is formed as assessed by cord number, cord length and branching points (nodes). Arborization increased with time and was maximal at day 4 (fig. 4); by day 6, the networks showed evidence of disintegration.

In order to further address the fine structure of the interactions of PCs with ECs in this 3-D culture system, we analyzed the matrices by transmission electron microscopy. Although some ECs could be unambiguously identified by the presence of Weibel-Palade bodies, other ECs could not, and thus, it was sometimes difficult to distinguish ECs from PCs in transmission electron micrographs. Therefore, to facilitate the analysis, we prelabeled the PCs with iron oxide nanoparticles before suspension in the matrix (fig. 5b). This did not impair PC viability or function, and transmission electron micrographs unambiguously indicated that investing PCs were able to form peg and socket junctions with ECs that had formed lumenized cords (fig. 5c) much as they do in vivo [14]. As electron microscopy revealed close EC-PC contact, we assessed if gap junctions could form between the ECs and PCs within the gel. Our laboratory has previously shown that BCECF-AM could be used to fluorescently label cy- 

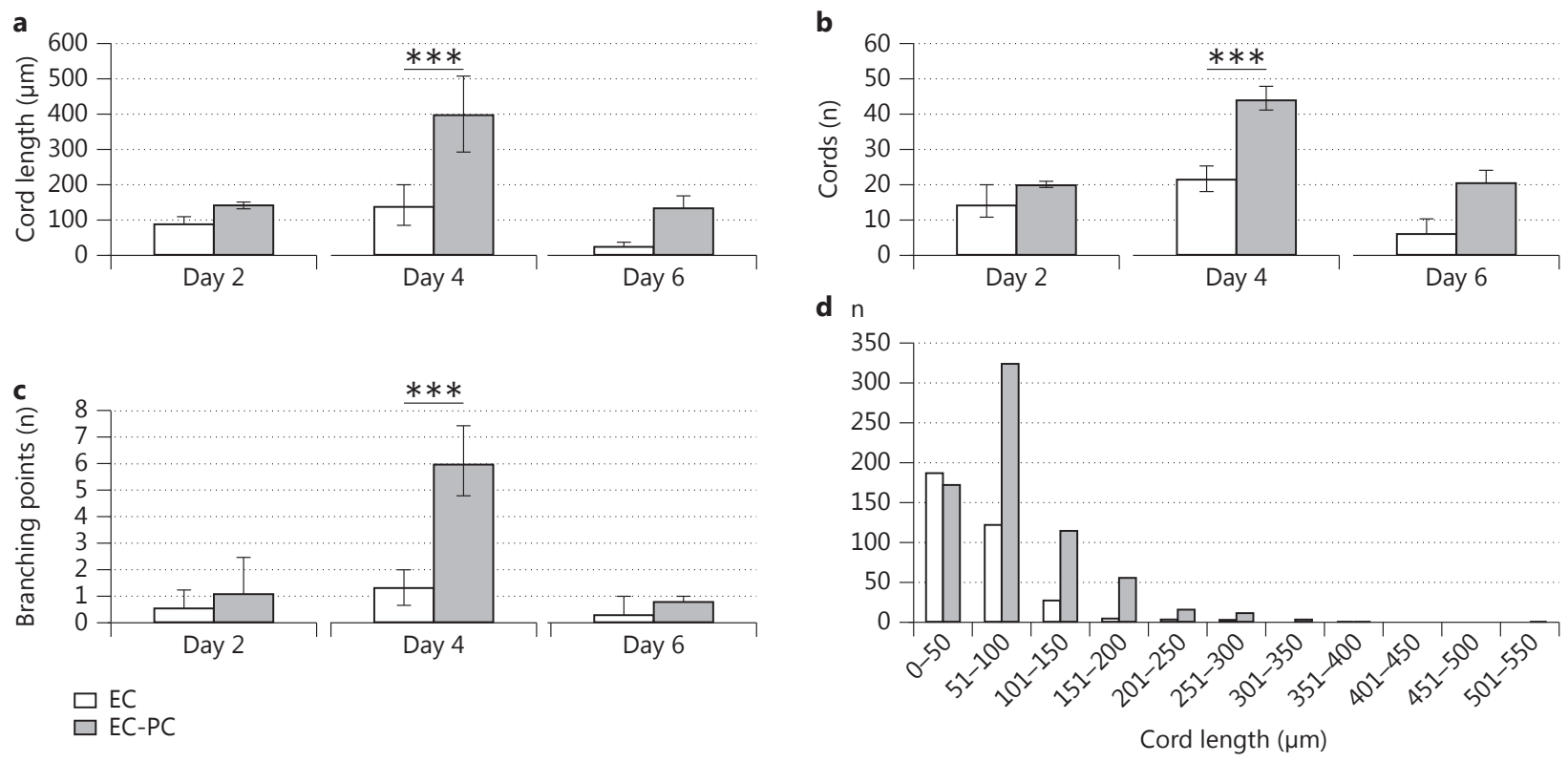

Fig. 4. Quantitative analysis of effects of $\mathrm{PC}$ coculture on cord formation by HUVEC in vitro. ${ }^{* * *} \mathrm{p}<0.001$. a Total cord length. $\mathbf{b}$ Cord number. $\mathbf{c}$ Number of branching points. $\mathbf{d}$ Cord length distribution showing increased arborization when cocultured with PCs at day $4(\mathrm{n}=3$; except on day $6, n=2)$.

toplasm of one cell type with the hydrolyzed product, BCECF [15]. If gap junctions are then formed with another cell type, labeled with a different fluor, BCECF will transfer to that cell and costaining will be seen. No transfer of fluorescent dye was observed in either direction (data not shown).

When HMVECs, microvascular ECs, were used in place of HUVECs in the gel, networks (fig. 6a) were formed which lumenized via vacuolization (fig. 6b). When cocultured with placental PCs, the lumens were again narrower over the time course of culture (fig. 6c). Interestingly, the diameters of HUVEC versus HMVEC cords were not different from each other in either monoculture or coculture with PCs.

\section{Discussion}

Microvascular stability depends upon PC investment of lumenized EC structures, and abnormal PC-EC interactions underlie a number of vascular disorders. Cocultures, including mixed monolayers, transwell geometries and agarose migration assays, have been used to study these interactions, but the ECs in such geometries do not form a lumenized vascular network. ECs plated on Matrigel $[16,17]$, a proangiogenic but incompletely defined tumor matrix that, unless depleted, contains a complex mixture of growth factors, form 'tubes' that rest on top of the gel rather than within a 3-D matrix. The effects of this system on PC behavior have not been reported. Here, we describe a simple system using a collagen/fibronectinbased gel to which other components can be added that allows better modeling of actual microvessel geometry over longer periods of time. In addition, the significant changes in gene expression that occur in the 3-D culture system compared to 2-D cultures may better reflect the in vivo setting [18-22].

Coculture of HUVECs with bovine retinal PCs has been achieved over a similar time course, but required treatment of ECs with phorbol esters or hematopoietic stem cell cytokines [23, 24]. Association of bovine retinal PCs with HUVEC cords was seen without matrix contraction, perhaps because human placental PCs are more contractile than bovine retinal PCs. The system we 

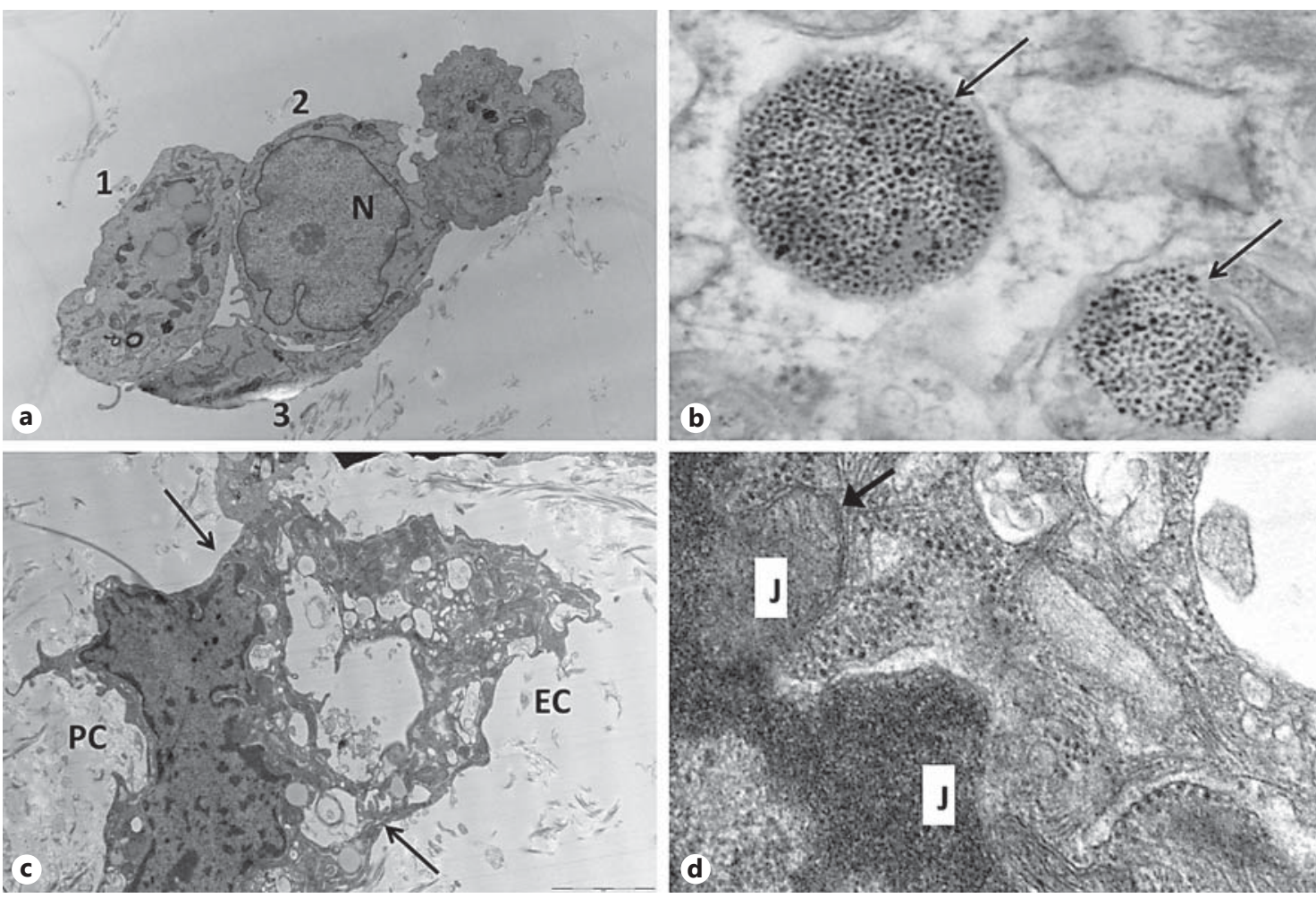

Fig. 5. Ultrastructural analysis of PC and EC interactions. Transmission electron microscopy. a Lumen formation resulting from cell-cell contact among three unlabeled cells (numbered as 1, 2 and 3). N = Nucleus. b Electron-dense iron oxide core of nanoparticles (arrows) internalized by PCs. c PC in contact (arrows) with HUVEC (EC) with lumen. d Higher-power view of two peg and socket junctions (J) between PC and HUVEC with a potential basement membrane (arrow).

describe allows cells with contractile force to be cocultured with ECs, and the modification of the ECs by Bcl2 transduction allows interactions to be assessed over a longer period, without the introduction of pharmacological agents. The rigidity given to the gel by the PGA mesh also aids manipulation of the gel for fixation and sectioning.

By observing the cells over a 7-day period, we found that PCs modify EC tubular networks, increasing arborization and preventing endothelial tubes from increasing in diameter. This effect of PCs is consistent with the vascular pathology seen in diseases with PC dropout such as diabetic retinopathy [1] and hereditary hemorrhagic telangiectasia [2] that are characterized by formation of ectatic naked endothelial vessels.

Although the transduction with Bcl-2 decreased the rate of apoptosis, we still observed disintegration of cords by 6 days. This loss of structures likely accounts for the decrease in arborization we observed from day 4 to day 6 . While it is known that primary vasculature assembled in vivo undergoes pruning as part of the maturation process, we hesitate to interpret the loss of cords as an example of this process and propose that our model be used to focus on processes that occur during the initial assembly of lumenized microvessels. The need to optimize the starting number of PCs in our system to prevent PCs from overgrowing ECs does add the disadvantage that different PC:EC ratios, as seen in different vascular beds, cannot be studied. Flow-mediated effects are not incorporated into this system either. Whilst there were peg and socket junctions formed between ECs and PCs, an electrical connection between the two cell types was not demonstrated. This, along with the nonuniform coverage of the EC networks by PCs, suggests there may be a secreted factor that maintains the narrow lumen rather than this effect being a contact-dependent process.

Others have presented in vitro models for the study of ECs and PCs. An elegant system using coculture of human brain vascular PCs in the collagen bulk of a microfluidic circuit formed by lithography has recently been 
Fig. 6. Lumen formation by HMVEC in 3-D matrix gels in vitro. Phase contrast micrographs through the 3-D matrix, without a PGA mesh, containing Bcl-2-transduced HMVEC only. a Low-power view of cord networks at $48 \mathrm{~h}$. b High-power view showing vacuoles (one labeled with an arrow) in cords at $24 \mathrm{~h}$. c Quantitative analysis of tube diameter $(\mathrm{n}=3$; $\mathrm{p}<0.01$ for each time point). Black line $=$ HMVEC only; dashed line = HMVEC and PC coculture. Error bars are standard deviations.

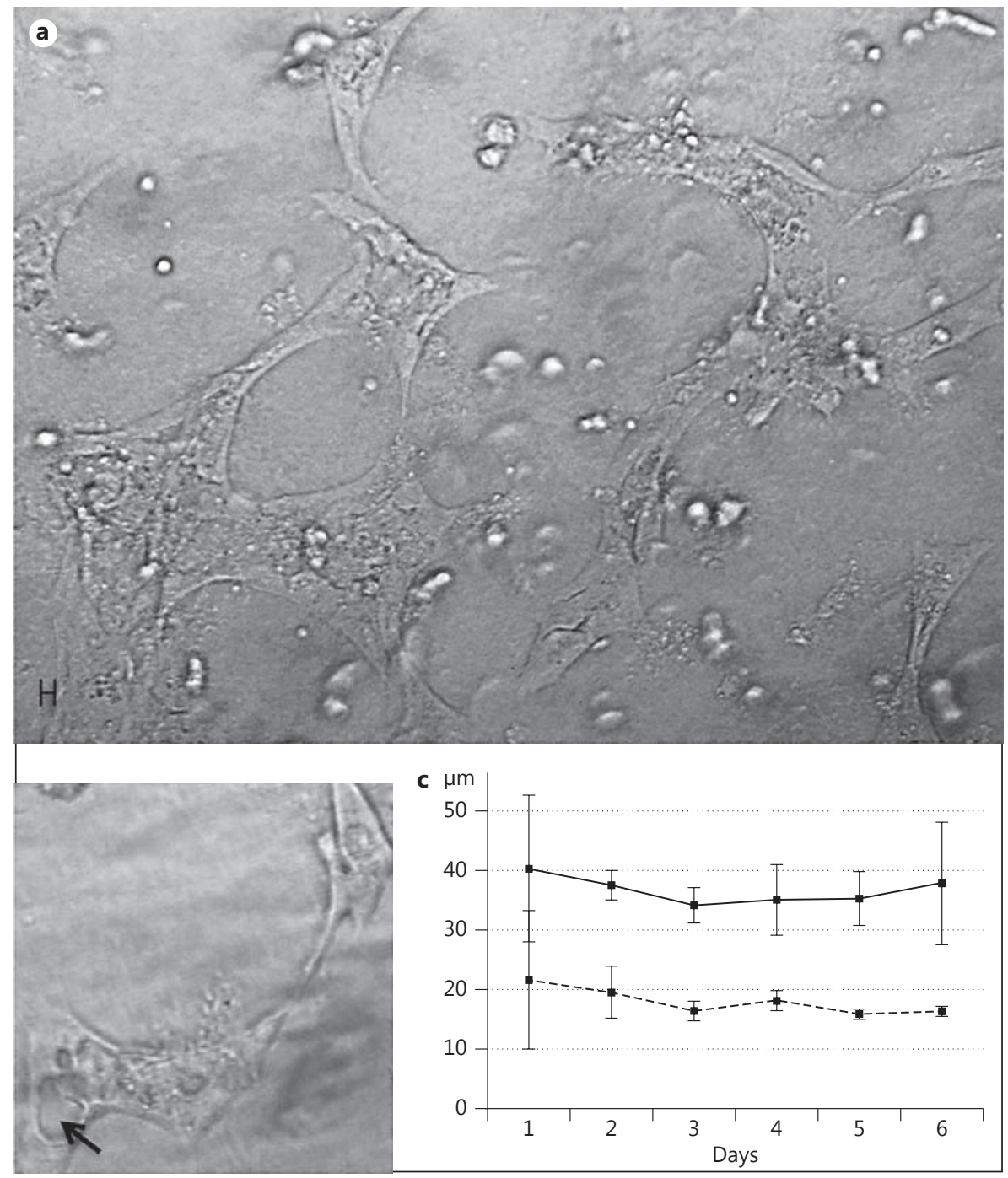

used to assess EC angiogenic sprouting [25]. The much simpler system we describe here uses a widely accessible supply of human vascular cells that behave in a consistent fashion yet still allows interactions between vascular cell types to be analyzed. The modifications we have introduced, particularly the reduction in EC apoptosis through $\mathrm{Bcl}-2$ transduction and the inhibition of gel contraction via PGA stabilization, should be broadly applicable to cells from tissue-specific vascular beds. We demonstrate that our simple system allows the study of interactions between PCs and either macrovascular and microvascular ECs. In summary, we have refined a simple collagenbased 3-D system to study the stabilizing interaction of ECs and PCs during vascular assembly for several days by preventing matrix contraction with a PGA mesh. This system will allow interrogation of the signaling between the two cell types and matrix-stimulated responses to be studied in greater detail in vitro.

\section{Acknowledgements}

We wish to acknowledge the funding of this work by National Institutes of Health grant HL-085416, Kidney Research UK grant TF13-2010 and the NIHR Cambridge Biomedical Research Centre. W.G. Chang is funded by the National Institutes of Health grant KL2 RR024138. 


\section{References}

1 Hammes HP: Pericytes and the pathogenesis of diabetic retinopathy. Horm Metab Res 2005;37(suppl 1):39-43.

-2 Lebrin F, Srun S, Raymond K, Martin S, van den Brink S, Freitas C, Breant C, Mathivet T, Larrivee B, Thomas JL, Arthur HM, Westermann CJ, Disch F, Mager JJ, Snijder RJ, Eichmann A, Mummery CL: Thalidomide stimulates vessel maturation and reduces epistaxis in individuals with hereditary hemorrhagic telangiectasia. Nat Med 2010;16:420-428.

-3 McCarty MF, Somcio RJ, Stoeltzing O, Wey J, Fan F, Liu W, Bucana C, Ellis LM: Overexpression of PDGF-BB decreases colorectal and pancreatic cancer growth by increasing tumor pericyte content. J Clin Invest 2007; 117:2114-2122.

4 Schlondorff D, Banas B: The mesangial cell revisited: no cell is an island. J Am Soc Nephrol 2009;20:1179-1187.

5 Schrimpf C, Duffield JS: Mechanisms of fibrosis: the role of the pericyte. Curr Opin Nephrol Hypertens 2011;20:297-305.

-6 Lopez-Novoa JM, Rodriguez-Pena AB, Ortiz A, Martinez-Salgado C, Lopez Hernandez FJ: Etiopathology of chronic tubular, glomerular and renovascular nephropathies: clinical implications. J Transl Med 2011;9:13.

7 Petrova TV, Karpanen T, Norrmen C, Mellor R, Tamakoshi T, Finegold D, Ferrell R, Kerjaschki D, Mortimer P, Yla-Herttuala S, Miura N, Alitalo K: Defective valves and abnormal mural cell recruitment underlie lymphatic vascular failure in lymphedema distichiasis. Nat Med 2004;10:974-981.

8 Shepherd BR, Jay SM, Saltzman WM, Tellides G, Pober IS: Human aortic smooth muscle cells promote arteriole formation by coengrafted endothelial cells. Tissue Eng Part A 2009; 15:165-173.

9 Gimbrone MA Jr: Culture of vascular endothelium. Prog Hemost Thromb 1976;3:1-28.
10 Maier CL, Shepherd BR, Yi T, Pober JS: Explant outgrowth, propagation and characterization of human pericytes. Microcirculation 2010;17:367-380.

11 Cheng EH, Kirsch DG, Clem RJ, Ravi R, Kas$\tan$ MB, Bedi A, Ueno K, Hardwick JM: Conversion of Bcl-2 to a Bax-like death effector by caspases. Science 1997;278:1966-1968.

12 Nelson GN, Roh JD, Mirensky TL, Wang Y, Yi T, Tellides G, Pober JS, Shakarin P, Shapiro EM, Saltzman WM, Papademetris X, Fahmy TM, Breuer CK: Initial evaluation of the use of USPIO cell labeling and noninvasive MR monitoring of human tissue-engineered vascular grafts in vivo. FASEB J 2008;22:38883895.

13 Harrington JK Chahboune H, Criscione JM, Li AY, Hibino N, Yi T, Villalona GA, Kobsa S, Meijas D, Duncan DR, Devine L, Papademetri X, Shin'oka T, Fahmy TM, Breuer CK: Determining the fate of seeded cells in venous tissue-engineered vascular grafts using serial MRI. FASEB J 2011;25:4150-4161.

14 Diaz-Flores L, Gutierrez R, Varela H, Rancel N, Valladares F: Microvascular pericytes: a review of their morphological and functional characteristics. Histol Histopathol 1991;6: 269-286.

15 Guinan EC, Smith BR, Davies PF, Pober JS: Cytoplasmic transfer between endothelium and lymphocytes: quantitation by flow cytometry. Am J Pathol 1988;132:406-409.

16 Nor JE, Peters MC, Christensen JB, Sutorik MM, Linn S, Khan MK, Addison CL, Mooney DJ, Polverini PJ: Engineering and characterization of functional human microvessels in immunodeficient mice. Lab Invest 2001;81: 453-463.

17 Skovseth DK, Yamanaka T, Brandtzaeg P, Butcher EC, Haraldsen G: Vascular morphogenesis and differentiation after adoptive transfer of human endothelial cells to immunodeficient mice. Am J Pathol 2002;160: 1629-1637.
18 Enis DR, Dunmore B, Johnson N, Pober JS, Print CG: Antiapoptotic activities of Bcl-2 correlate with vascular maturation and transcriptional modulation of human endothelial cells. Endothelium 2008;15:59-71.

19 Harma V, Virtanen J, Makela R, Happonen A, Mpindi JP, Knuuttila M, Kohonen P, Lotjonen J, Kallioniemi O, Nees M: A comprehensive panel of three-dimensional models for studies of prostate cancer growth, invasion and drug responses. PLoS One 2010; 5:e10431.

20 Ghosh S, Spagnoli GC, Martin I, Ploegert S, Demougin P, Heberer M, Reschner A: Threedimensional culture of melanoma cells profoundly affects gene expression profile: a high density oligonucleotide array study. J Cell Physiol 2005;204:522-531.

21 Delcommenne M, Streuli CH: Control of integrin expression by extracellular matrix. J Biol Chem 1995;270:26794-26801.

22 Martin KJ, Patrick DR, Bissell MJ, Fournier MV: Prognostic breast cancer signature identified from 3D culture model accurately predicts clinical outcome across independent datasets. PLoS One 2008;3:e2994.

23 Stratman AN, Malotte KM, Mahan RD, Davis MJ, Davis GE: Pericyte recruitment during vasculogenic tube assembly stimulates endothelial basement membrane matrix formation. Blood 2009;114:5091-5101.

24 Stratman AN, Davis MJ, Davis GE: VEGF and FGF prime vascular tube morphogenesis and sprouting directed by hematopoietic stem cell cytokines. Blood 2011;117:3709-3719.

25 Zheng Y, Chen J, Craven M, Choi NW, Totorica S, Diaz-Santana A, Kermani P, Hempstead B, Fischbach-Teschl C, Lopez JA, Stroock AD: In vitro microvessels for the study of angiogenesis and thrombosis. Proc Natl Acad Sci USA 2012;109:9342-9347. 